\title{
Características Físico- Químicas de Queijo Minas Frescal Comercializados na Cidade de Anápolis-Go
}

\author{
Judith L. Xavier, Kelly N. Marra, Natan L. Ribeiro \\ \& Thábita E. D. Mota
}

O queijo minas frescal é um produto obtido por coagulação enzimática do leite e classificado como um queijo fresco. Neste contexto foram realizadas analises físicoquímicas de lipídios, acidez, alcalinidade das cinzas, $\mathrm{pH}$, umidade e cloretos em cinco amostras de queijo branco de diferentes cidades de Anápolis- Goiás, as cinco amostras analisadas foram inspecionadas pela Inspeção do estado- Sistema EIS. Foi feita a comparação entre os valores achados e os permitidos pela legislação. Foi detectado que dentre as amostras estudadas, somente a análise das cinzas obteve resultado satisfatório em todas as amostras. E somente a análise de lipídios cumpriu o s padrões

Palavras Chave: Queijo Minas Frescal; Qualidade; Características Físico-Químicas.

The Minas's Frescal Cheese is the product obtained by enzymatic coagulation of milk and it is classified as fresh cheese. In this context held physic-chemical analysis of lipids, acidity, alkalinity of ash, $\mathrm{pH}$, moisture and chlorides in five samples from fresh white cheese, from different brands sold in supermarkets in the city of Anápolis-Goiás, the five samples analyzed all were inspected by the State Inspection - EIS System. The comparison of the values found with the values allowed by the legislation was held. Was detected in the study that among the five samples studied, the ash analysis was the only that was satisfactory in all samples. The analysis of lipids found only one brand has met the standards.

Keywords: Mina's Frescal Cheese; Quality; Physic-Chemical Characteristics. 


\section{Introdução}

O leite é uma bebida bastante importante para o ser humano, sendo o primeiro alimento que consumimos e nos acompanhando até a vida adulta. As proteínas são um dos principais constituintes do leite, por possuir um alto valor nutritivo, e por fornecer nitrogênio e aminoácidos essenciais para o ser humano ${ }^{1}$. As principais proteínas constituintes são a caseína e as proteínas do soro, dentre elas, a $\beta$-lactoglobulina, $\alpha$ - lactoalbumina, soroalbumina bovina e imunoglobulinas.

Outros componentes importantes são os lipídeos, compostos por $98 \%$ apolares, sendo eles, glicerídeos (triglicerídios, diglicerídios e monoglicerídios), e ácidos graxos livres, e outros $2 \%$ lipídeos polares1. A lactose é o açúcar mais simples e o mais constante em proporção no leite, além da glicose e galactose. O leite contém entre 45 e $50 \mathrm{~g} /$ litro de lactose $\mathrm{e}^{2,3}$.

Entre os sais minerais encontram-se principalmente, cobre, ferro, boro, manganês, zinco, iodo, entre outros. As enzimas presentes no leite são encontradas em pequenas concentrações, entre elas estão as lípases, as proteases, a fosfatase, a oxidase, a catalase, a superóxido dismutase,a sulfidroxilase e a transferase ${ }^{5}$.

As vitaminas lipossolúveis presentes no leite são as vitaminas $\mathrm{A}, \mathrm{D}$ e $\mathrm{E}$, e as hidrossolúveis presentes principalmente, no soro do leite, podendo assim ser isoladas. Em geral tem-se riboflavina (vitamina B2), vitamina B12, tiamina (vitamina B1), vitamina A e vitamina $C$, nos quais os teores variam em função do tratamento térmico aplicado ao produto $^{2}$.

Dentre os produtos feitos a base de leite o queijo Minas Frescal é um dos mais populares. Para o seu preparo devese utilizar leite integral de boa qualidade, coado, pasteurizado (aquecido a uma temperatura de $65{ }^{\circ} \mathrm{C}$ por 30 minutos) e resfriado em água corrente a $38^{\circ} \mathrm{C} 4$. Para sua produção em escala industrial é feito um processo de acidificação seguida pela adição de coalho, agente que promove a coagulação do leite, formando a massa do queijo. Esse método é chamado de "coagulação enzimática", pois o coagulante é formado por uma enzima, que é uma proteína com propriedades específicas ${ }^{4}$.
Após a adição dos ingredientes ao leite ocorre a coagulação em aproximadamente 40 minutos, o que se detecta com um corte da coalhada em cubos de $2 \mathrm{~cm}$ com o auxílio de uma faca ou liras. Isto possibilita que o soro seja drenado dos pedaços individuais de coalhada ${ }^{4,5}$.

Após o corte é realizada a mexedura, o queijo tem como característica principal o alto teor de umidade. Portanto, a mexedura deve ser realizada de tal forma que essa característica seja preservada. Ela consiste na agitação dos cubos durante 1 minuto e, em seguida, deixá-los em repouso durante 3 minutos. Repete-se a operação por 30 minutos.

Em seguida realiza-se a enformagem para dar ao queijo sua forma característica. Seguindo essa etapa é realizada então a salga. A maioria dos queijos é salgado por imersão em salmoura ou pela aplicação de sal seco na superfície. Esta é a última etapa de produção, promovendo a sinérese de modo sutil. No entanto, não é um método satisfatório para o controle da umidade da coalhada. A umidade pode ser melhor assegurada garantindo que o grau de acidificação, aquecimento e mexedura no tanque sejam adequados para a variedade do queijo ${ }^{4,5}$.

O queijo Minas Frescal é classificado como semigordo, com teor de gordura que varia de 25 a 44,9\%. A gordura existente exerce uma função importante na cor, na consistência e no sabor final do queijo. O sal que contribui entre 1,4 e 1,6\% é utilizado na fabricação de queijos com várias finalidades. Contribui com o sabor, melhora a textura, aparência do queijo, controla a fermentação lática determinando o nível correto de acidez, inibe o desenvolvimento de microrganismos indesejáveis e auxilia na expulsão do soro ${ }^{6}$.

\section{Metodologia}

Foram analisadas 5 amostras de Queijo Minas Frescal, sendo elas de 5 marcas diferentes, tituladas como marcas " $\mathrm{A}$ ", "B", "C", "D" e "E". Coletadas em supermercados da cidade de Anápolis - GO. As amostras de queijo foram submetidas às seguintes análises físico-químicas: lipídeos, acidez titulável, alcalinidade das cinzas, $\mathrm{pH}$, umidade e cloretos. Todas as análises foram realizadas em triplicata, no laboratório de 
Físico-Química da Universidade Estadual de Goiás.

Para a Análise de Lipídeos foi utilizado o método de extração de Soxlet. A amostra foi aquecida em um forno a uma temperatura de $105^{\circ} \mathrm{C}$ por 1 hora e depois colocada no dessecador por 30 minutos. Foi feito um cartucho de papel com o fundo forrado de algodão onde foi inserido aproximadamente 5,00g de alimento. O refluxo foi realizado utilizando hexano durante 2 horas, a $25^{\circ} \mathrm{C}$. O cartucho foi suspendido por mais 2 hora, vedado e seco em uma estufa a $105^{\circ} \mathrm{C}$ por 1 hora. Resfriou-se em um dessecador e pesouse a amostra ${ }^{7}$.

Para a determinação de acidez foram transferidos $10 \mathrm{~g}$ da amostra para um béquer de $150 \mathrm{~mL}$, acrescentado cerca de $50 \mathrm{~mL}$ de água morna destilada, e levada ao ultrassom até a possível dissolução. Foi transferido quantitativamente para balão volumétrico de $100 \mathrm{~mL}$, esfriado em água corrente e completado o volume. Transferiu-se uma alíquota de 50 $\mathrm{mL}$ para um erlenmeyer de $250 \mathrm{~mL}$, acrescentado 10 gotas de solução alcoólica de fenolftaleína a $1 \%$ e titulado com solução de hidróxido de sódio $0,1 \mathrm{~N}$ até leve coloração rósea persistente por aproximadamente 30 segundos ${ }^{8,9}$

Para a análise de cinzas foram submetidas $5,0 \mathrm{~g}$ de amostra a incineração através de bico de bunsen numa placa aquecedora. Foi transferido quantitativamente as cinzas, obtidas na metodologia de resíduo mineral fixo, para béquer de $400 \mathrm{~mL}$, usando pequenas porções de água destilada até $75 \mathrm{~mL}$. Adicionou-se aos poucos $50 \mathrm{~mL}$ de solução de ácido clorídrico $0,1 \mathrm{~N}$, e triturou-se as cinzas com um bastão de vidro e foi transferido eventuais restos da amostra, juntamente com o ácido para um béquer de 400 $\mathrm{mL}$. Lavado com água destilada o bastão e o cadinho.

$\mathrm{O}$ béquer foi coberto com um vidro de relógio e levado à ebulição moderada por 5 minutos, e depois resfriado. Adicionou-se $30 \mathrm{~mL}$ de solução de cloreto de cálcio a $40 \%$.

Foi deixado em repouso por 10 minutos e adicionado 10 gotas de solução alcoólica de fenolftaleína a $1 \%$ e titulado o excesso de ácido clorídrico com solução padronizada de hidróxido de sódio $0,1 \mathrm{~N}$ até que fosse obtida turvação e coloração rósea persistente. ${ }^{9,10}$.

A medida do $\mathrm{pH}$ foi realizada utilizando um $\mathrm{pHmetro}$ calibrado com soluções tampões pH 4 e 7 . Adicionado cerca de $20 \mathrm{~mL}$ de água em um béquer de $50 \mathrm{~mL}$. Foi acrescentado cerca de $5 \mathrm{~g}$ de amostra previamente preparada, misturando com bastão de vidro de modo a obter uma pasta homogênea; Em seguida levada ao ultrassom, e depois da amostra homogeneizada foi medido o $\mathrm{pH}$ das amostras em triplicata ${ }^{7}$.

A umidade e sólidos totais foi medida através de um cadinho de porcelana, cerca de $5 \mathrm{~g}$ da amostra em estufa a aproximadamente $102^{\circ} \mathrm{C}$ durante 1 hora. Esfriado em dessecador e pesado. Pesado a amostra preparada e homogeneizada e levada novamente à estufa. Esfriado em dessecador e pesado. Repetido até que a massa permaneceu constante. As operações de pesagem foram feitas o mais rápido possível e a secagem foi conduzida sem que houvesse escurecimento da amostra ${ }^{10}$.

Para a análise do cloreto foi utilizado o método potenciometrico, para isso pesado em um béquer exatamente cerca de $5 \mathrm{~g}$ de amostra preparada. Adicionado $30 \mathrm{~mL}$ de água a $50^{\circ} \mathrm{C}$ e homogeneizado com o misturador. Lavouse o misturador com $10 \mathrm{~mL}$ de água coletando o lavado no béquer. Foi adicionado cerca 2 a $3 \mathrm{~mL}$ da solução de ácido nítrico $4 \mathrm{~N}$ e colocado o eletrodo do potenciômetro na suspensão. Titulando o conteúdo do béquer com a solução de nitrato de prata $0,1 \mathrm{~N}$ agitando continuamente até quase alcançar o ponto final. Em seguida, foi titulado cuidadosamente até atingir o ponto final, que corresponde a máxima diferença de potencial observada entre duas idênticas adições sucessivas (aproximadamente 0,05 mL) da solução de nitrato de prata 0,1 N9.

\section{Resultados e Discussão}

Os resultados das análises feitas podem ser visualizados abaixo nas tabelas de 1 a 6 . Os parâmetros utilizados para determinar o nível de qualidade foram determinados pelo Ministério da Agricultura ou comparados com a literatura conhecida. O queijo Minas Frescal deve seguir os seguintes parâmetros: um índice lipídico de 25 a 44\%; um teor de cinzas entre 0,7 e 6,0\%; e índice de umidade acima de $55 \%$. Os parâmetros não citados não são determinados pelo Ministério da Agricultura ${ }^{10}$.

Em relação a acidez titulavel foi usado al encontrada 
por Marques e Oliveira11 de $0,052 \%$ como parâmetro. Estudos de Ferreira6 que determina que um $\mathrm{pH}$ entre 6,3 e 6,7 para queijo Minas. Além disso ele também determina que o índice de cloreto deve ser entre 1,4\% e 1,6\%

Comparando os resultados observados aos esperados percebe-se que o queijo "E" é o mais adequado em relação ao índice de lipídios, sendo que, todos os outros possuem uma quantidade abaixo do esperado. Nenhum dos queijos possuía um índice de acidez titulavel satisfatório, porém a alcalinidade das cinzas de todos estava de acordo com o esperado. Em relação ao $\mathrm{pH}$ nenhum deles apresentou um valor ideal, sendo os queijos "A", "D" e "E" acima do esperado e os queijos "B e "C" abaixo. O índice de umidade de todos os queijos apresentou valores abaixo de $55 \%$. E o índice de cloreto também não foi satisfatório uma vez que os queijos "A" e "C" obtiveram valores acima do esperado e o restante um valor abaixo.

Tabela 1. Média percentual dos valores de Lipídeos obtidos de Queijo Minas Frescal comercializados em supermercados da cidade de Anápolis-GO

\begin{tabular}{|c|c|c|}
\hline AMOSTRA & LIPÍDEOS (\%) & $\begin{array}{c}\text { DESVIO PADR ̃̃O } \\
(\Theta)\end{array}$ \\
\hline A & 4 & $\pm 0,153$ \\
\hline B & 22,7 & $\pm 0,101$ \\
\hline C & 15,8 & $\pm 0,208$ \\
\hline D & 19,7 & $\pm 0,200$ \\
\hline E & 29 & $\pm 0,643$ \\
\hline
\end{tabular}

Tabela 2. Média percentual dos valores de acidez Titulável obtidos de Queijo Minas Frescal comercializados em supermercados da cidade de Anápolis-GO

\begin{tabular}{|c|c|c|}
\hline AMOSTRA & ACIDEZ $(\%)$ & $\begin{array}{c}\text { DESVIO PADRÃO } \\
(\Theta)\end{array}$ \\
\hline A & 0,097 & $\pm 0,002$ \\
\hline B & 0,503 & $\pm 0,015$ \\
\hline C & 0,459 & $\pm 0,014$ \\
\hline D & 0,11 & $\pm 0,021$ \\
\hline E & 0,141 & $\pm 0,002$ \\
\hline
\end{tabular}

Tabela 3. Média percentual dos valores de alcalinidade das cinzas obtidos de Queijo Minas Frescal comercializados em supermercados da cidade de Anápolis-GO

\begin{tabular}{|c|c|c|}
\hline AMOSTRA & $\begin{array}{c}\text { ALCALINIDADE } \\
\text { DAS CINZAS (\%) }\end{array}$ & $\begin{array}{c}\text { DESVIO PADRÃO } \\
(\Theta)\end{array}$ \\
\hline A & 2,83 & $\pm 0,100$ \\
\hline B & 4,99 & $\pm 0,061$ \\
\hline C & 2,97 & $\pm 0,096$ \\
\hline D & 3,49 & $\pm 0,042$ \\
\hline E & 2,75 & $\pm 0,084$ \\
\hline
\end{tabular}

Tabela 4. Média percentual dos valores de $\mathrm{pH}$ obtidos de Queijo Minas Frescal comercializados em supermercados da cidade de Anápolis-GO

\begin{tabular}{|c|c|c|}
\hline AMOSTRA & $\mathrm{pH}$ & $\begin{array}{c}\text { DESVIO PADRÃO } \\
(\Theta)\end{array}$ \\
\hline $\mathrm{A}$ & 6,88 & $\pm 0,005$ \\
\hline $\mathrm{B}$ & 5,18 & $\pm 0,006$ \\
\hline $\mathrm{C}$ & 5,59 & $\pm 0,010$ \\
\hline $\mathrm{D}$ & 6,83 & $\pm 0,006$ \\
\hline $\mathrm{E}$ & 6,76 & $\pm 0,005$ \\
\hline
\end{tabular}

Tabela 5. Média percentual dos valores de umidade obtidos de Queijo Minas Frescal comercializados em supermercados da cidade de Anápolis-GO

\begin{tabular}{|c|c|c|}
\hline AMOSTRA & UMIDADE (\%) & $\begin{array}{c}\text { DESVIO PADRÃO } \\
(\Theta)\end{array}$ \\
\hline A & 48,7 & $\pm 0,404$ \\
\hline B & 46 & $\pm 7,05$ \\
\hline C & 44,4 & $\pm 2,83$ \\
\hline D & 40,4 & $\pm 2,05$ \\
\hline E & 39,7 & $\pm 3,907$ \\
\hline
\end{tabular}

Tabela 6. Média percentual dos valores de Cloretos obtidos de Queijo Minas Frescal comercializados em supermercados da cidade de Anápolis-GO

\begin{tabular}{|c|c|c|}
\hline AMOSTRA & CLORETOS (\%) & $\begin{array}{c}\text { DESVIO PADRÃO } \\
(\Theta)\end{array}$ \\
\hline A & 0,962 & $\pm 0,061$ \\
\hline B & 2,064 & $\pm 0,275$ \\
\hline C & 1,204 & $\pm 0,140$ \\
\hline D & 1,878 & $\pm 0,010$ \\
\hline E & 1,789 & \pm 0.153 \\
\hline
\end{tabular}




\section{Conclusões}

Nota-se que pela falta de padronização na formulação de queijo, foram encontradas em algumas análises, certas variações de resultados, em produtos com os mesmos ingredientes descritos nos rótulos, mas de marcas diferentes.

É muito importante que sejam levadas em consideração as condições higiênico-sanitárias na fabricação e armazenamento do produto, visto que essas interferem diretamente na qualidade final do queijo.

Nas análises de lipídeos somente a marca "E" atendeu aos padrões. Nas análises de cinzas todas as amostras atenderam satisfatoriamente aos padrões esperados segundo a legislação. E nas análises de acidez, pH, umidade e cloretos, nenhuma das marcas atendeu satisfatoriamente ao esperado.

Portanto, torna-se necessário a padronização do produto e maior fiscalização dos produtores industriais, visto que as amostras são todas de marcas registradas no SIE.

\section{Agradecimentos}

Agradeço a professora Kelly N Marra, a UEG e a todos que me deram apoio por essa oportunidade.

\section{Referências Bibliográficas}

1. Neto, R. J., Avaliação Tecnológica e físico-quimica de queijo tipo Minas Frescal obtido a partir do leite concentrado por Ultrafiltração. 2006. 66. Dissertação (Pós Graduação em Engenharia de Alimentos) - Universidade Federal de Santa Catarina, Florianópolis, Santa Catarina, 2006.

2. Ordóñez, J. A.; Díaz, O.; Cobos, A.; Hoz, L. Tecnologia de alimentos - Alimentos de origem animal. Vol. 2. Tradução: Fátima Murad. Porto Alegre: Artmed, 2005.

3. Fenaille, F.; Parisod, V.; Visani, P.; Populaire, S. ; Tabet, J. C. ; Guy, P. A. Modifications of milk constituents during processing: A preliminary benchmarking study. International. Dairy Journal, v. 16, p. 728-739, 2006

4. EMBRAPA. Tecnologia de Fabricação do Queijo Minas Frescal. Luiz Carlos Vieira; José de Brito Lourenço Júnior.

5. Dagostin, J. L. A. Avaliação de atributos Microbiológicos e físicoquímicos de queijo minas frescal elaborado a partir de queijo carbonatado. (2011) Dissertação de Mestrado UFPR.
6. Machado, E. C.; Ferreira, C. L. L. F.; Fonseca, L. M; Soares, F. M.; Queijo artesanal produzido na região do Serro, Minas Gerais. Ciência e Tecnologia de Alimentos, Campinas, v. 24, n. 4, p. 516521, out/dez, 2004.

7. IAL, 2008, Instituto Adolfo Lutz (São Paulo). Métodos físicoquímicos para análise de alimentos/coordenadores Odair Zenebon, Neus Sadocco Pascuet e Paulo Tiglea -- São Paulo: Instituto Adolfo Lutz, 2008

8. Merck. Reactivos, diagnóstica, productos químicos 1992/93. Darmstadt, 1993. 1584 p. Disponível em: < www.jusbrasil.com.br/ diarios/532120/dou-secao-1-02-05-2003-pg-9>

9. Brasil. Ministério da Agricultura. Secretaria Nacional de Defesa Agropecuária. Laboratório Nacional de Referência Animal. Leite fluido. Métodos analíticos oficiais para controle de produtos de origem animal e seus ingredientes: métodos físicos e químicos. v. II, cap. 14, p. 13-14. Brasília, DF, 1981

10. Brasil. Instrução Normativa ${ }^{\circ} 68$ de 12/12/2006 / MAPA Ministério da Agricultura, Pecuária e Abastecimento (D.O.U. 14/12/2006).

11. Marques, M.C.; Oliveira, C.A.F. Avaliação das características físicoquímicas do queijo Minas frescal produzido com leite contendo diferentes níveis de células somáticas. Pirassununga: FZEA/USP, 2004. 15p. (Iniciação Científica)

\section{Judith L. Xavier, Kelly N. Marra*, Natan L. Ribeiro \& Thábita E. D. Mota}

Universidade Estadual de Goiás - Campus de Ciências Exatas e Tecnológicas, Br-153, 3105 - Fazenda Barreiro do Meio, 75.132-903, Anápolis - Goiás - Brasil;

*E-mail: kelly.nobre@ueg.br 